\title{
In Vivo Olfactory Model of APP-Induced Neurodegeneration Reveals a Reversible Cell-Autonomous Function
}

\author{
Ning Cheng, ${ }^{1}$ Huaibin Cai, ${ }^{2}$ and Leonardo Belluscio ${ }^{1}$ \\ ${ }^{1}$ Developmental Neural Plasticity Unit, National Institute of Neurological Disorders and Stroke, and ${ }^{2}$ Laboratory of Neurogenetics, National Institute of \\ Aging, National Institutes of Health, Bethesda Maryland 20892
}

\begin{abstract}
Amyloid precursor protein (APP) has long been linked to the neurodegeneration of Alzheimer's disease (AD), but the associated cell death has been difficult to capture in vivo, and the role of APP in effecting neuron loss is still unclear. Olfactory dysfunction is an early symptom of $\mathrm{AD}$ with amyloid pathology in the olfactory epithelium correlating well to the brain pathology of AD patients. As olfactory sensory neurons (OSNs) regenerate continuously with immature and mature OSNs coexisting in the same olfactory epithelium, we sought to use this unique system to study APP-induced neurodegeneration. Here we have developed an olfactory-based transgenic mouse model that overexpresses humanized APP containing familial AD mutations (hAPP) in either mature or immature OSNs, and found that despite the absence of extracellular plaques a striking number of apoptotic neurons were detected by 3 weeks of age. Importantly, apoptosis was restricted to the specific population overexpressing hAPP, either mature or immature OSNs, sparing those without hAPP. Interestingly, we observed that this widespread neurodegeneration could be rapidly rescued by reducing hAPP expression levels in immature neurons. Together, these data argue that overexpressing hAPP alone could induce cell-autonomous apoptosis in both mature and immature neurons, challenging the notion that amyloid plaques are necessary for neurodegeneration. Furthermore, we show that hAPP-induced neurodegeneration is reversible, suggesting that $\mathrm{AD}$-related neural loss could potentially be rescued. Thus, we propose that this unique in vivo model will not only help determine the mechanisms underlying AD-related neurodegeneration but also serve as a platform to test possible treatments.
\end{abstract}

\section{Introduction}

Alzheimer's disease (AD) is the most common neurodegenerative disorder yet has no effective treatments. A pathological hallmark of $\mathrm{AD}$ is extracellular plaques containing amyloid- $\beta(\mathrm{A} \beta)$ peptides derived from amyloid precursor protein (APP). As genetic studies have also linked APP mutations to familial forms of $\mathrm{AD}$, an amyloid hypothesis has been formulated that plaques cause the large-scale neuronal loss typical of late-stage $\mathrm{AD}$ patients (Hardy and Selkoe, 2002). While this provides a framework for AD-related studies, it remains controversial, as the density of amyloid deposits does not correlate with the degree of cognitive impairment observed in patients (Hardy and Selkoe, 2002). Transgenic studies overexpressing APP have also provided in vivo evidence that pathological and functional changes occur before plaque pathology, including inhibition of the ubiquit-

Received April 3, 2011; revised July 7, 2011; accepted July 13, 2011.

Author contributions: N.C.,H.C., and L.B. designed research; N.C. performed research; N.C. and L.B. analyzed data; N.C. and L.B. wrote the paper.

This work was supported by the NINDS intramural program, project number 1ZIANS003116-01. We thank Li Bai and Rini Singh for assistance with genotyping and immunohistochemistry. We thank the members of the Belluscio laboratory for constructive comments on this work. We thank the NINDS EM facility for assistance with the electron microscopy studies.

The authors declare no competing financial interests.

Correspondence should be addressed to Dr. Leonardo Belluscio, Developmental Neural Plasticity Unit, National Institute of Neurological Disorders and Stroke, National Institutes of Health, 35 Convent Drive, Bethesda, MD 20892. E-mail: belluscl@ninds.nih.gov.

DOI:10.1523/JNEUROSCI.1714-11.2011

Copyright $\odot 2011$ the authors $\quad 0270-6474 / 11 / 3113699-06 \$ 15.00 / 0$ in-proteasome system, mitochondrial defects, synaptic dysfunction and cognitive deficits (Wirths et al., 2004; Gouras et al., 2005; LaFerla et al., 2007; Bayer and Wirths, 2010; Tampellini and Gouras, 2010). Interestingly, these pathologies have largely been attributed to intracellular $A \beta$ accumulation, although the existence of low-level extracellular $A \beta$ is difficult to completely rule out (Oddo et al., 2003). Unfortunately, most transgenic models poorly mimic the neurodegeneration of AD patients, such that APP mutations alone are sufficient to cause extensive neural loss in humans, but not in these models. For example, although APP23 mice show a modest loss of cortical and hippocampal neurons, it is far less than the loss observed in AD patients, while other models must involve additional disease-related proteins, and extensive aging, to produce neuron loss (Duyckaerts et al., 2008; Elder et al., 2010; Wirths and Bayer, 2010). Thus, while APP has been implicated in effecting neurodegeneration, its role is poorly understood.

Olfactory dysfunction is one of the earliest symptoms of $\mathrm{AD}$, often preceding cognitive deficits by many years (Bacon et al., 1998; Hawkes, 2003). Studies have shown that amyloid pathology in the olfactory epithelium (OE) parallels brain pathology (Arnold et al., 2010) along with neurodegeneration in the $\mathrm{OE}$ of $\mathrm{AD}$ patients (Talamo et al., 1989). We hypothesized that olfactory sensory neurons (OSNs) may be more sensitive to AD-related factors than cortical neurons and thus serve as a model to study APP-induced neurodegeneration. Since OSNs regenerate naturally from basal stem cells in the OE producing immature OSNs that migrate toward the apical surface as they mature (Farbman, 

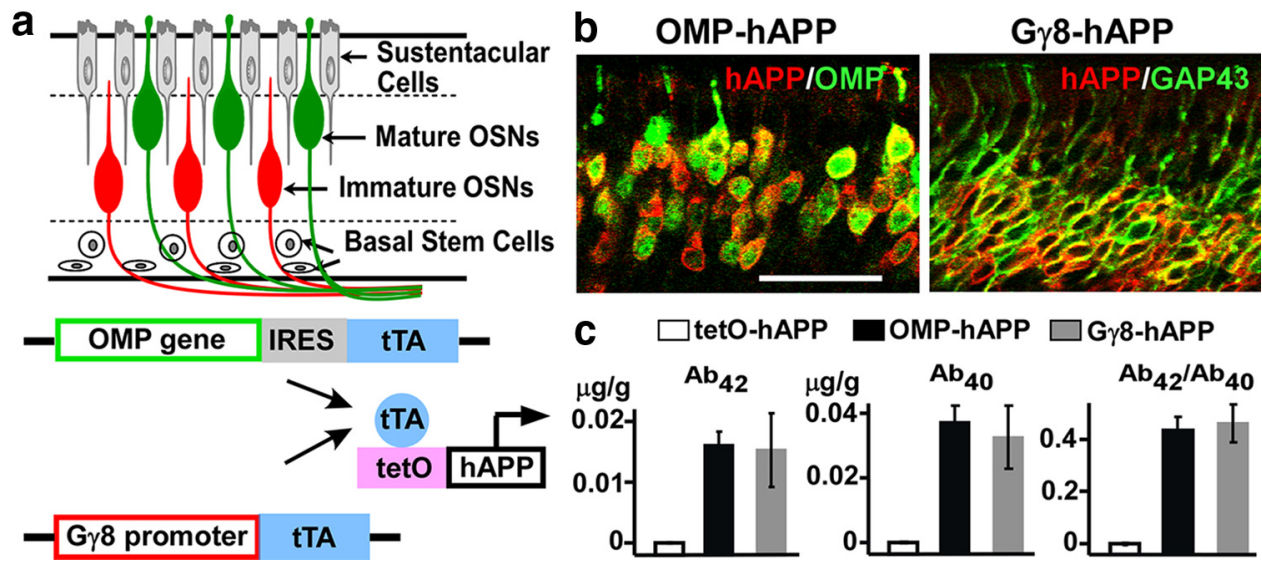

Figure 1. Selectively overexpressing hAPP in either mature or immature OSNs. $\boldsymbol{a}$, Schematic for generating transgenic lines that selectively overexpress hAPP in either mature (OMP promoter, OMP-hAPP line) or immature ( $\mathrm{G} \gamma 8$ promoter, G $\gamma 8$-hAPP line) OSNs (see Materials and Methods). $\boldsymbol{b}$, Colocalization of hAPP protein (red) with OMP protein (green) in OMP-hAPP line (left), and with GAP43 protein (green) in $G \gamma 8$ - hAPP line (right). Scale bar, $40 \mu \mathrm{m}$. c, Overexpression of human $A \beta_{42}, A \beta_{40}$ in $0 M P$ - $h A P P$ line $\left(A \beta_{42}, 0.016 \pm 0.002 ; A \beta_{40}, 0.037 \pm 0.005 \mu \mathrm{g} / \mathrm{g} ; \mathrm{rati0}, 0.44 \pm 0.05\right.$; $n=5)$, and $G \gamma 8$-hAPP line $\left(A \beta_{42}, 0.015 \pm 0.006 ; A \beta_{40}, 0.033 \pm 0.010 \mu \mathrm{g} / \mathrm{g} ;\right.$ ratio, $\left.0.46 \pm 0.07 ; n=5\right)$, compared with control, tet0-hAPP line $\left(A \beta_{42}, 0 ; A \beta_{40}, 0 \mu \mathrm{g} / \mathrm{g} ; n=3\right)$. Values are mean \pm SD. Animals: $3-4$ weeks old.

1990; Calof et al., 1996; Cummings and Belluscio, 2008), the OE provides a continuous source of developing neurons. Here we have engineered two in vivo transgenic lines, which selectively overexpress a mutated humanized APP (hAPP) in either mature or immature OSNs, in a temporally controllable manner. We show that largescale cell-autonomous neuronal death was readily and directly observed in OSNs, and could be rescued by turning off hAPP expression.

\section{Materials and Methods}

Transgenic lines. We used the Tetracycline-(tet-off)-transactivation system (tTA/tetO), allowing spatial-temporal control of transgene expression with the addition of doxycycline (Dox) turning-off promotor function (Gossen and Bujard, 1992; Lewandoski, 2001). TetO- $h A P P$ line contains the $h A P P$ transgene (humanized $\mathrm{A} \beta$-domain with familial $\mathrm{AD}$ mutations-KM570, 571NL 'Swedish' and V617F 'Indiana') (Jankowsky et al., 2005). OMP-tTA and Gy8-tTA lines express the tetracycline transactivator in either mature or immature OSNs, respectively (Nguyen et al., 2007). Each tTA-containing line was crossed with tetO-hAPP line to generate lines that selectively express hAPP in either mature OSNs driven by the OMP promoter (OMP-hAPP line), or the immature OSNs driven by the G $\gamma 8$ promoter ( $G \gamma 8$-hAPP line). Presumably, hAPP expression begins during embryonic development when the OMP and $\mathrm{G} \gamma 8$ promoters are activated (Nguyen et al., 2007). Genotyping was performed to recognize mutants containing both the $t T A$ - and tet $O$-transgenes. Littermates containing only tet $O-h A P P$ transgene were selected as controls and both sexes used for experiments. All mice were mixed $(129 \times$ C57BL/6) background.

PCR primers used for genotyping (forward, reverse): OMP-tTA, $5^{\prime}$ GGTTGCGTATTGGAAGATCAAGAGC-3', 5' -GAGGAGCAGCTAGAAGAATGTCCC $33^{\prime} ; \quad G \gamma 8-t T A, \quad 5^{\prime}$-GTTCCAGCCCCCAGTCCACACTCC-3' ${ }^{\prime}, 5^{\prime}$-CATGTCCAGATCGAAATCGTCTAGC- ${ }^{\prime}$; tet $O-h A P P$, 5' -CCGAGATCTCTGAAGTGAAGATGGATG-3', 5' -CCAAGCCTAGACCACGAGAATGC-3'.

ELISA of $A \beta$. Colorimetric sandwich ELISA kits with antibodies against human $\mathrm{A} \beta 40$ and $\mathrm{A} \beta 42$ (Invitrogen) were used. Acutely dissected OE tissue was homogenized and centrifuged. Supernatant was loaded on ELISA plate. Assay was performed according to manufacturer's manual with all standards and samples measured in duplicate.

Immunohistochemistry. Fluorescence immunohistochemistry on $\mathrm{OE}$ sections was performed as previously described (Cummings and Belluscio, 2010). Primary antibodies: APP, 1:1000; (6E10, Covance); OMP,1: 5000 (Wako); GAP43,1:1000 (Novus Biologicals); cleaved-caspase3, 1:1000 (Cell Signaling Technology), Sections were examined using confocal microscopy (LSM510, Zeiss).
Immunoelectron microscopy. Previously established protocol (TaoCheng et al., 2006) was followed. OMP-hAPP mice were perfused transcardially with 2\% PFA and 2\% glutaraldehyde in $0.1 \mathrm{M}$ PBS. Coronal sections of OE were incubated with an antibody against human APP (6E10, 1:11,000) followed by a Nanogold-conjugated secondary antibody (1:250; Nanoprobes). After dehydration, silver enhancement and staining with $2 \%$ uranyl acetate, tissue was embedded and sectioned, and examined with an electron microscope (1200EX II; JEOL).

BrdU pulse injection. BrdU (50 mg/kg body weight, Sigma) in $0.9 \%$ $\mathrm{NaCl}$ was intraperitoneal injected into 2-week-old mice. Later olfactory tissue was collected and sections were incubated in $2 \mathrm{~N} \mathrm{HCl}$ at $37^{\circ} \mathrm{C}$ for 20 min to denature the DNA, before immunohistochemical staining using a BrdU antibody (1:500; Accurate Chemical).

Turning off hAPP expression. Doxycycline-containing chow (Doxchow; $6 \mathrm{~g} / \mathrm{kg}, 0.5$ inch pellets, Bio-Serv) was fed to lactating mothers when $G \gamma 8$ - $h A P P$ pups were 2 to 3 weeks old, and directly to OMP-hAPP mice from 3 to 4 weeks of age after weaning. Dox prevents the tTA protein from binding to the tetO-sequence.

Cell counting. OMP-, GAP43-, caspase3- or BrdU-positive cells in the septal OE spanning dorsal to ventral zones were counted manually using signal intensity and size threshold. Images of four to six sample sections were taken from each animal representing the anterior, middle, and posterior parts of the turbinates.

Statistical analysis. Student's $t$ test was performed to test statistical significance, assuming two-tailed distribution and two-sample unequal variance. All values are mean $\pm \mathrm{SD} ;{ }^{*} p<0.05$ and ${ }^{* *} p<0.001$ in all figures.

\section{Results}

Selective overexpression of hAPP in two OSN populations Studies have shown that specific molecular markers can be used to distinguish and manipulate mature versus immature OSNs: olfactory marker protein (OMP) for mature OSNs and G-protein gamma-subunit $8(\mathrm{G} \gamma 8)$ or growth-associated protein 43 (GAP43) for immature OSNs (Verhaagen et al., 1989; Nguyen et al., 2007). Using these markers and the tetracycline-transactivation system we developed two in vivo transgenic lines that selectively express hAPP in either mature OSNs (OMP-hAPP line) or immature OSNs ( $G \gamma 8$ hAPP line) (Fig. 1a; Materials and Methods). This approach produced clear and specific hAPP overexpression in both mutant lines by 3 weeks of age that predominantly colocalized with OMP in the $O M P-h A P P$ line and with GAP43 in the G $\gamma 8$-hAPP line (Fig. $1 b$ ). Total APP protein in the mutants was $\sim 2 \times$ endogenous levels (data 

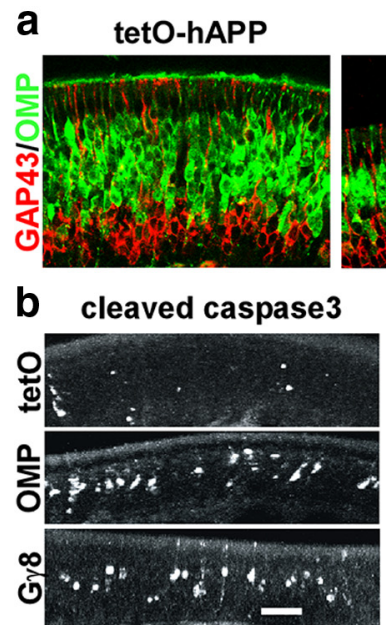

OMP-hAPP
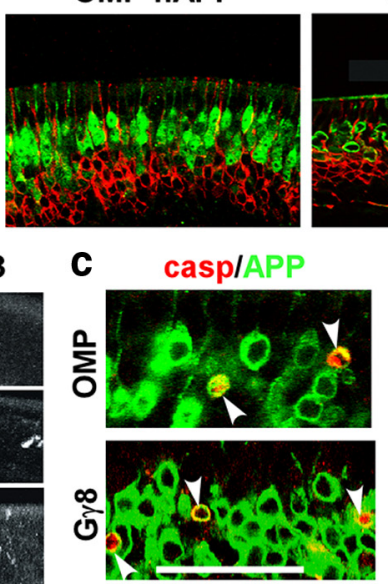

Gr8-hAPP

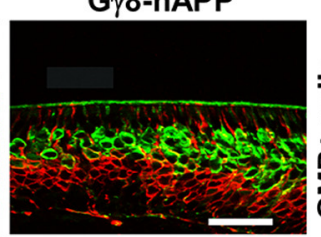

d
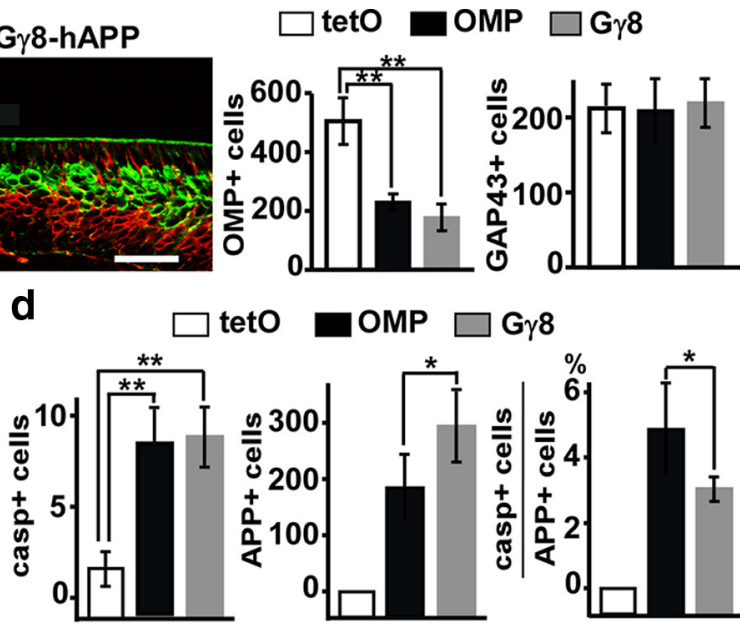

tetO

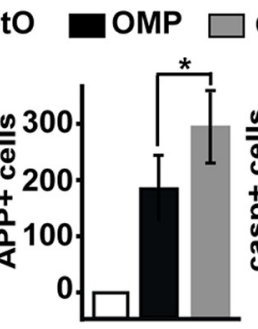

Figure 2. Rapid and widespread apoptosis in OSNs expressing hAPP. $\boldsymbol{a}$, Mature OSN numbers (OMP-positive; green) were significantly smaller in both OMP- $h A P P(230 \pm 26, n=5)$ and G $\gamma 8-h A P P(177 \pm 41, n=4)$ than tet0-hAPP $(505 \pm 77, n=6)$, while immature OSNs (GAP43-positive; red) remained similar: OMP-hAPP (209 $\pm 42, n=4), G \gamma 8-h A P P(219 \pm 31, n=4)$, tet0-hAPP $(212 \pm 31, n=4) \cdot \boldsymbol{b}$, Increased cleaved-caspase3 expression in mutant OE (white dots, collapsed confocal stack). c, Colocalization of cleaved-caspase3 (red) and hAPP (green) in both

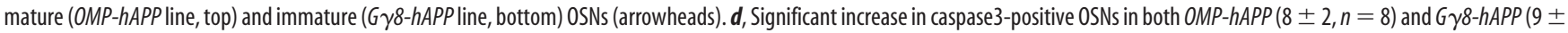
$2, n=4)$, compared with tet0-hAPP $(2 \pm 1, n=7)$. However, there were fewer hAPP-expressing cells and a greater ratio of caspase3:hAPP-positive cells in OMP-hAPP ( $h A P P, 184 \pm 58$; ratio, $4.9 \pm$ $1.4 \%$ ) than in $G \gamma 8-h A P P(h A P P, 294 \pm 62$; ratio, $3.0 \pm 0.3 \%)$. Scale bars, $40 \mu \mathrm{m}$. Values are mean \pm SD per mm 0 E, single optical slice. Significance: ${ }^{*} p<0.05{ }^{* *} p<0.001$. Animals: $3-4$ weeks old.

not shown). By comparison, transgene-derived $\mathrm{A} \beta_{42}$ levels were $\sim 25 \%$ of those reported in 4-week-old CaMKII-hAPP mice, which use a similar tTA-activation strategy, while $\mathrm{A} \beta_{40}$ levels were similar (Jankowsky et al., 2005). Interestingly, both $\mathrm{A} \beta_{42}$ and $\mathrm{A} \beta_{40}$ levels, as well as the ratio between the two were comparable to those in the well noted "triple-transgenic" $\mathrm{AD}$ mouse at 5-7 months of age (Oddo et al., 2003) (Fig. 1c).

Rapid and widespread apoptosis in OSNs expressing hAPP Immunohistochemical analysis revealed that in both of our mutant lines at 3 weeks of age, the OEs were thinner than in controls, and mature (OMP-positive) OSNs were significantly reduced while the immature (GAP43-positive) population did not change (Fig. 2a). Control mice had similar composition of mature and immature OSNs in the OE as previously reported (Vedin et al., 2009; Macdonald et al., 2010). To determine whether reduced mature-OSN population was due to elevated neuron death via apoptosis, as proposed by earlier studies in $\mathrm{AD}$ patients ( $\mathrm{Su}$ et al., 1994; Troncoso et al., 1996), we performed cleaved-caspase3 immunohistochemical staining on $\mathrm{OE}$ sections. We found a striking increase in caspase3-positive cells in both mutant lines (Fig. 2b), which was approximately four times the control level (Fig. $2 d$ ). Importantly, caspase 3 signal visibly colocalized with hAPPexpressing neurons (Fig. $2 c$ ) and did not occur in the neighboring population in the OE that did not express hAPP. For example, increased caspase 3 signal was absent in the immature OSNs in the $O M P$ - $h A P P$ line, and absent in the mature OSNs in the Gy8$h A P P$ line. Elevated apoptosis was further supported by TUNEL staining and condensed nuclear morphology that often colocalized with caspase 3 signal (data not shown). Together, these data clearly demonstrated that hAPP overexpression alone was sufficient to induce neurodegeneration of OSNs through apoptosis. Notably, the ratio of caspase3-positive cells to hAPP-positive cells was greater in the OMP-hAPP line than in the Gy8-hAPP line (Fig. $2 d$ ), suggesting that mature OSNs may be more vulnerable to hAPP-induced apoptosis than the immature ones.
Cell-autonomous hAPP-induced ultrastructural disruption of the OE

To determine whether hAPP-induced neurodegeneration was a consequence of extracellular plaques as proposed by the amyloid hypothesis, we examined the hAPP immunohistochemical signal in 3-week-old mutant mice using an antibody that recognizes both the full-length protein and the $\mathrm{A} \beta$ peptide. Our data revealed clear intracellular signal but no evidence of aggregated extracellular signal (Fig. 3a). Using immuno-electron microscopy on OMP-hAPP OE, we further showed that hAPP labeling was predominantly confined to the nuclear-envelope, the lumen of the endoplasmic reticulum, and the plasma membrane facing the extracellular space, consistent with the intracellular trafficking route of APP (Thinakaran and Koo, 2008) (Fig. 3b). However, no aggregated extracellular gold particles were detected. We also observed broad ultrastructural changes in $O M P-h A P P O E$ such as fewer dendritic knobs with fewer cilia, consistent with fewer mature OSNs (Reese, 1965; Schwob et al., 1992), and large numbers of apoptotic bodies (Fig. $3 c-f$ ).

Both mature and immature hAPP-expressing OSNs showed reduced survival but differential capacity for rescue

To determine at which stage of their life cycle OSNs died, we performed in vivo bromodeoxyuridine (BrdU) injections and collected $\mathrm{OE}$ tissue at different times postinjection. Earlier studies showed that immature OSNs reach maturity 6-7 d after their final division, thus defining a distinct transition point (Schwob et al., 1992; Carr and Farbman, 1993). In the G $\gamma 8$-hAPP line, most BrdU-labeled neurons disappeared before $7 \mathrm{~d}$ of age, consistent with the described immature time-window, whereas in the OMP$h A P P$ line, most BrdU-labeled neurons were lost between 7 and $14 \mathrm{~d}$ of age (Fig. 4a,b). These data indicated that mature and immature OSNs were both susceptible to hAPP-induced cell death. Interestingly, in the $G \gamma 8$ - $h A P P$ line, basal stem cell proliferation was upregulated, as indicated by twice as many BrdUlabeled cells $2 \mathrm{~h}$ postinjection compared with OMP-hAPP line and control, which had similar level of proliferation (Vedin et al., 2009; Macdonald et al., 2010). This difference could explain why the GAP43-positive population remained constant in G $\gamma 8$ - $h A P P$ 
line despite the death of immature neurons and the depletion of mature OSNs (Fig. 2a).

Since expression of hAPP could be temporally controlled (see Materials and Methods), we next sought to determine whether the course of neuron loss could be reversed by shutting off the transgene. Mice were fed Dox-chow during the period when most BrdU-labeled cells disappeared in each mutant line (Fig. 4c). Dox treatment did not alter OSN survival in control mice (based on quantification of surviving BrdU-labeled cells, as well as caspase 3- and OMP-positive cells in the OE, data not shown), yet successfully turned off hAPP expression in both mutant lines within 1 week (hAPP immunohistochemical signal was reduced to background level, data not shown). Our data showed that significantly more BrdU-labeled cells survived in the Doxtreated than in the untreated $G \gamma 8$-hAPP $\mathrm{OE}$. There were also significantly fewer caspase3-positive cells and more OMPpositive cells in the Dox-treated $G \gamma 8$ $h A P P$ OE. By contrast, Dox treatment in the OMP-hAPP OE showed only modest improvements in caspase3- and OMPpositive cells (Fig. 4d). Thus our data showed that hAPP-induced neurodegeneration could be reversed in vivo.

\section{Discussion}

This study presents a new in vivo model that allows direct and rapid visualization of hAPP-induced neuron death. Our data demonstrate that hAPP overexpression alone was sufficient to induce neuronal death, directly linking hAPP to the cause of neurodegeneration. Here we used an APP that contains two familial ADmutations facilitating $\mathrm{A} \beta$ generation, but it will be interesting to determine whether both wild-type APP and other mutant forms resistant to secretase cleavage can also induce similar levels of apoptosis in OSNs. Since the cortical and hippocampal neural pathology in $\mathrm{AD}$ patients proceeds slowly, only a limited number of dying neurons can be detected at any one time, making it difficult to study the neurodegenerative process with most animals models (Yuan and Yankner, 2000). In this study, we show that in the olfactory system high levels of hAPP cause neurodegeneration via widespread apoptosis by 3 weeks of age, suggesting that susceptibility to hAPP-induced neurodegeneration might be linked to neuronal turnover. This may explain why regions that show high levels of neurogenesis, i.e., olfactory bulb (OB) and hippocampus, might also degenerate more rapidly (Hawkes, 2003; Takahashi et al., 2010). Interestingly, a recent study found that in mice overexpressing a mutated form of human-APP under the control of the prion-protein gene promoter (Tg2576 AD mice), the earliest amyloid deposits occur in the olfactory bulb, particularly the glomerular layer where OSNs project (Wesson et al., 2010). Since OSNs also express prion-protein, it is possible
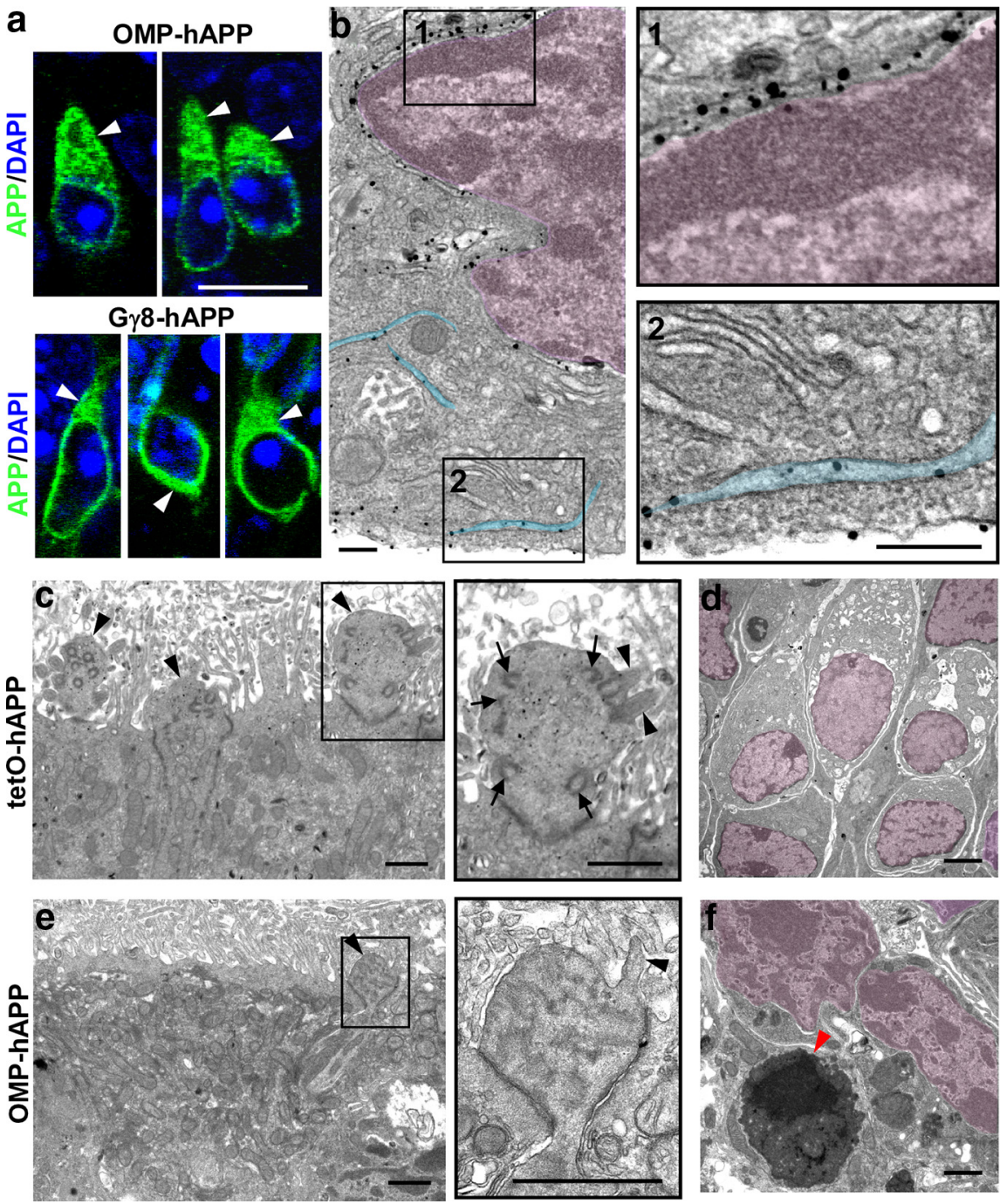

Figure 3. Cell-autonomous hAPP-induced ultrastructural disruption of the OE. $\boldsymbol{a}$, hAPP immunostaining showed intracellular (blue) lumen. $\boldsymbol{c}, \boldsymbol{d}$, EM images of control tet0-hAPP OE. c, Well-formed OSN dendritic knobs (left, arrowheads), of OMP-hAPP OE. $\boldsymbol{e}$, Very few smaller dendritic knobs (left, arrowhead) with less cilia (right, arrowhead). $f$, Disorganized OSN cell bodies around an apoptotic nucleus (red arrowhead). Scale bars: $\boldsymbol{a}, 10 \mu \mathrm{m} ; \boldsymbol{b}, 200 \mathrm{~nm} ; \boldsymbol{c}-\boldsymbol{f}, 1 \mu \mathrm{m}$. Animals: 3- 4 weeks old.

that the OE of Tg2576 mice might also be degenerated thus contributing to the observed olfactory dysfunction. Similarly, we also noted extensive changes in the OB circuitry and impairment in odor detection in our system (data not shown).

Although both mature and immature OSNs were susceptible to hAPP-induced death, mature OSNs expressing hAPP had a higher percentage of apoptotic cells than immature OSNs. In addition, our data showed that hAPP-induced neurodegeneration could be rapidly reversed by shutting off hAPP expression in immature OSNs but more slowly in mature ones. The reason for this observed difference is not yet clear. Differential requirements for survival of mature versus immature OSNs, including neurotrophin dependence, neurotrophin receptor expression, odorevoked activity, and turnover rate (Carson et al., 2005), could all potentially contribute to the observed difference. Likewise, extensive studies exploiting $\mathrm{OB}$ removal and lesion have shown that mature and immature OSNs have different vulnerability to target-dependent apoptosis (Schwob et al., 1992; Carson et al., 
a

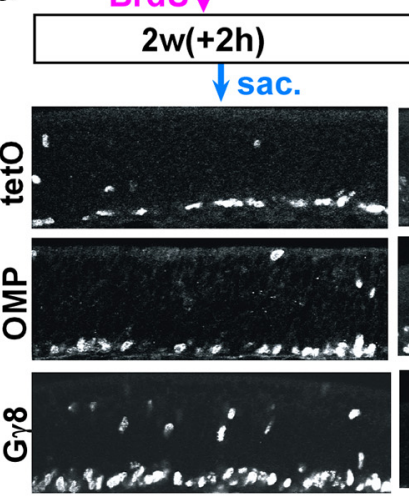

b

d
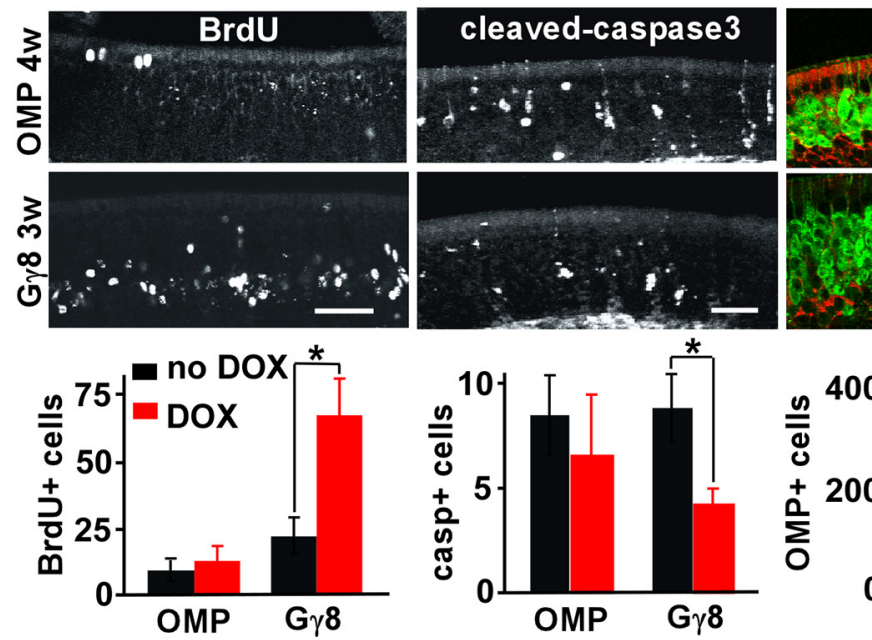

C
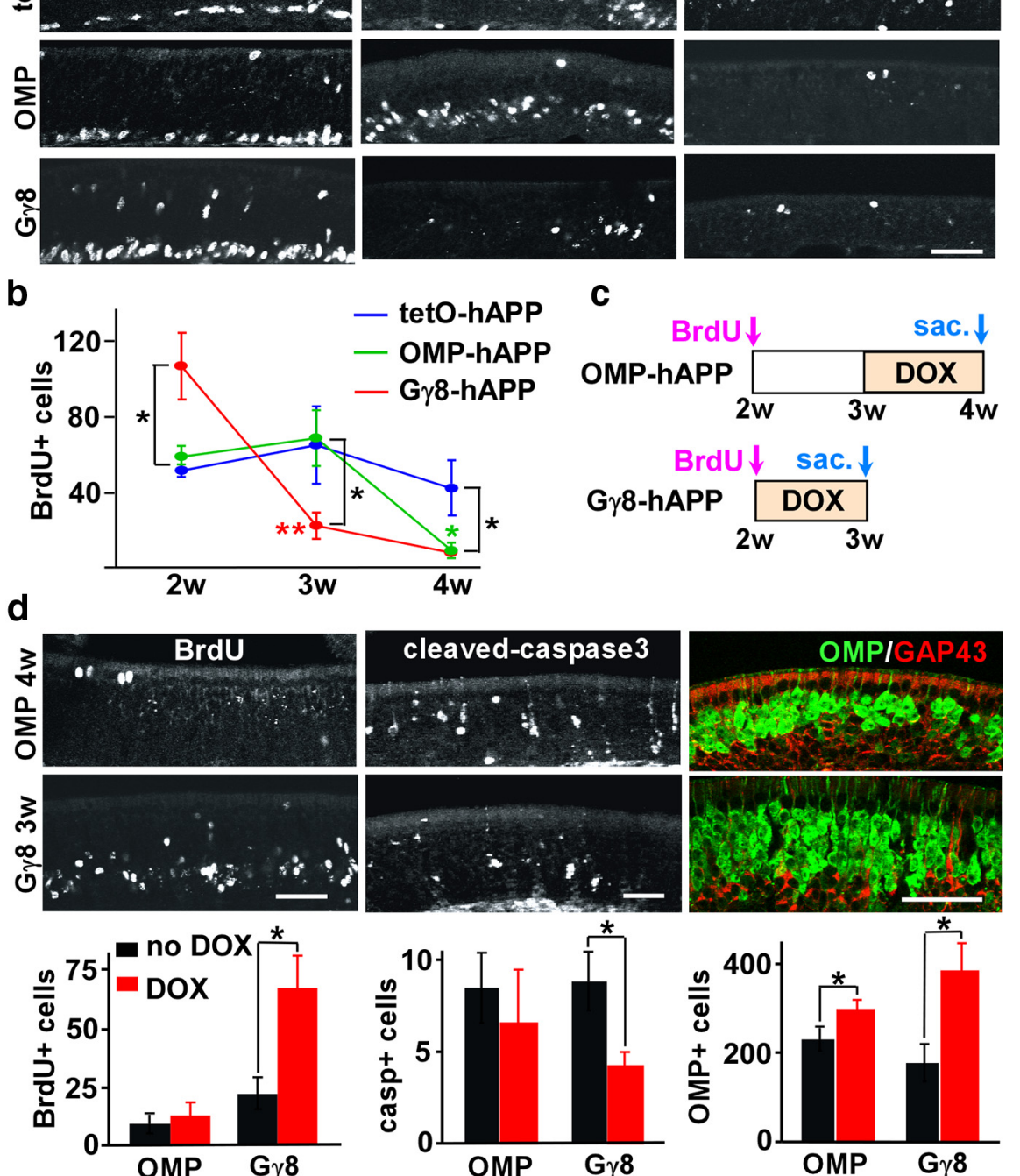

Figure 4. Both mature and immature hAPP-expressing OSNs showed reduced survival but were differentially rescued by turning off $h A P P$ transgene. $\boldsymbol{a}$, Experimental timeline shows all BrdU injections in 2-week-old animals and olfactory tissue harvested $2 \mathrm{~h}, 7 \mathrm{~d}$, or $14 \mathrm{~d}$ later. Images show OE immunostained for BrdU (white dots) corresponding to time points above. $\boldsymbol{b}$, Quantification of BrdU-positive cells at 2 week $(+2 \mathrm{~h})$ point showed a significantly greater number in G $\gamma 8$-hAPP line (107 \pm 18 , $n=5)$ than either OMP-hAPP (59 $\pm 6, n=3)$ or tet0-hAPP controls $(52 \pm 3, n=4)$ (black asterisk), revealing increased proliferation in $G \gamma 8$ - $h A P P$ line. A significant drop in BrdU-positive cells in 3-week-old G $\gamma 8$ - $h A P P$ mice $(22 \pm 7, n=4)$ compared with that in 2-week-old G $\gamma 8$-hAPP mice (red asterisks), as well as 3-week-old OMP- $h A P P(69 \pm 15, n=4)$ and tet0-hAPP $(65 \pm$ $21, n=5$ ) (black asterisk) identified a susceptibility period of apoptosis in immature OSNs. In OMP-hAPP mice, a similar significant drop occurred between 3 and 4 weeks of age ( $4 w: 9 \pm 4, n=4$; green asterisk), revealing a later susceptibility period in mature

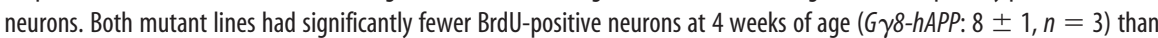
tet0-hAPP (43 $\pm 16, n=4$ ) (black asterisk). c, Timeline of recovery experiments shows BrdU injections in 2-week-old mice, followed by Dox-chow (which turns off hAPP expression) given to G $\gamma 8$-hAPP mice from 2 to 3 weeks of age, and OMP-hAPP mice from 3 to 4 weeks of age (see Materials and Methods), based upon the susceptibility periods for each line determined above. $d, 0 E$ from Dox-treated mice described in c, showing rapid recovery in G $\gamma 8$-hAPP line and delayed recovery in OMP-hAPP line. Graphs show significant changes in Dox-treated compared with nontreated G $\gamma 8$-hAPP OE, including increased BrdU-positive cells (Dox, $67 \pm 14, n=3$; no-Dox, $22 \pm 7, n=4)$; decreased caspase3-positive cells (Dox, $4 \pm 1, n=3$; no-Dox, $9 \pm 2, n=4$ ) and increased OMP-positive cells (Dox, $383 \pm 62, n=3$; no-Dox, $177 \pm 41, n=4$ ). Dox-treated OMP-hAPP mice showed smaller changes compared with nontreated in BrdU-positive cells (Dox, $13 \pm 5, n=3$; no-Dox, $9 \pm 4, n=4$ ), caspase3-positive cells (Dox, $7 \pm 3, n=3 ;$ no-Dox, $8 \pm 2, n=8$ ) and OMP-positive cells (Dox, $296 \pm 22, n=3 ;$ no-Dox, $230 \pm 26, n=5$ ). Significance: ${ }^{*} p<0.05 ;{ }^{* *} p<0.001$. Scale bar, $50 \mu \mathrm{m}$.
2005). Nevertheless, our BrdU experiments in immature OSNs show that initiating hAPP expression does not have to lead to cell death, suggesting it may be possible to rescue neurons undergoing hAPP-induced neurodegeneration. Moreover, early intervention may be a critical factor in determining recovery rate. Interestingly, previous studies have shown that reducing BACE1 levels can reduce degeneration of septohippocampal cholinergic neurons and memory deficits in 5XFAD mice, but the effect diminish with age, also supporting the importance of early intervention (Devi and Ohno, 2010).

Together, our data argue that in OSNs hAPP-induced neuron death is through a cell-autonomous mechanism. First, extracellular plaques were not observed when widespread apoptosis was readily present in our model. Second, in both $O M P-h A P P$ and G $\gamma 8$ - $h A P P$ lines, dying cells were confined to the OSN population that overexpressed hAPP, sparing the nonexpressing OSNs that reside side by side in the OE. Finally, neuron loss in this model could be rapidly reversed by turning off hAPP expression for merely a week, while extracellular amyloid deposits have been shown to be highly stable in vivo and can persist as long as 6 months after APP overexpression is turned off (Jankowsky et al., 2005).

Earlier studies showed that in $\mathrm{AD}$ patients, there is a marked decrease in mature OSNs compared with the normal OE (Talamo et al., 1989). Deficits in odor detection and discrimination are among the earliest symptoms of $\mathrm{AD}$ and loss of mature OSNs could certainly contribute to the pathologies. Our model mimics mature-OSN reduction reported in ADpatients and suggests that the neurodegenerative process is similar to that in other brain regions. Since the OE is readily accessible for both biopsy and delivering biochemical compounds, we propose that this model will prove valuable not only for studying the pathological process of $\mathrm{AD}$ but also for developing diagnostics tools and determining the efficacy of potential therapeutics.

\section{References}

Arnold SE, Lee EB, Moberg PJ, Stutzbach L, Kazi H, Han LY, Lee VM, Trojanowski JQ (2010) Olfactory epithelium amyloid-beta and paired helical filament-tau pathology in Alzheimer disease. Ann Neurol 67:462-469.

Bacon AW, Bondi MW, Salmon DP, Murphy C (1998) Very early changes in olfactory functioning due to Alzheimer's disease and the role of apolipoprotein E in olfaction. Ann N Y Acad Sci 855:723-731. 
Bayer TA, Wirths O (2010) Intracellular accumulation of amyloid-Beta-a predictor for synaptic dysfunction and neuron loss in Alzheimer's disease. Front Aging Neurosci 2:8.

Calof AL, Hagiwara N, Holcomb JD, Mumm JS, Shou J (1996) Neurogenesis and cell death in olfactory epithelium. J Neurobiol 30:67-81.

Carr VM, Farbman AI (1993) The dynamics of cell death in the olfactory epithelium. Exp Neurol 124:308-314.

Carson C, Saleh M, Fung FW, Nicholson DW, Roskams AJ (2005) Axonal dynactin p150Glued transports caspase- 8 to drive retrograde olfactory receptor neuron apoptosis. J Neurosci 25:6092-6104.

Cummings DM, Belluscio L (2008) Charting plasticity in the regenerating maps of the mammalian olfactory bulb. Neuroscientist 14:251-263.

Cummings DM, Belluscio L (2010) Continuous neural plasticity in the olfactory intrabulbar circuitry. J Neurosci 30:9172-9180.

Devi L, Ohno M (2010) Phospho-eIF2alpha level is important for determining abilities of BACE1 reduction to rescue cholinergic neurodegeneration and memory defects in 5XFAD mice. PLoS One 5:e12974.

Duyckaerts C, Potier MC, Delatour B (2008) Alzheimer disease models and human neuropathology: similarities and differences. Acta Neuropathol 115:5-38.

Elder GA, Gama Sosa MA, De Gasperi R (2010) Transgenic mouse models of Alzheimer's disease. Mt Sinai J Med 77:69-81.

Farbman AI (1990) Olfactory neurogenesis: genetic or environmental controls? Trends Neurosci 13:362-365.

Gossen M, Bujard H (1992) Tight control of gene expression in mammalian cells by tetracycline-responsive promoters. Proc Natl Acad Sci U S A 89:5547-5551.

Gouras GK, Almeida CG, Takahashi RH (2005) Intraneuronal Abeta accumulation and origin of plaques in Alzheimer's disease. Neurobiol Aging 26:1235-1244.

Hardy J, Selkoe DJ (2002) The amyloid hypothesis of Alzheimer's disease: progress and problems on the road to therapeutics. Science 297:353-356.

Hawkes C (2003) Olfaction in neurodegenerative disorder. Mov Disord $18: 364-372$.

Jankowsky JL, Slunt HH, Gonzales V, Savonenko AV, Wen JC, Jenkins NA, Copeland NG, Younkin LH, Lester HA, Younkin SG, Borchelt DR (2005) Persistent amyloidosis following suppression of Abeta production in a transgenic model of Alzheimer disease. PLoS Med 2:e355.

LaFerla FM, Green KN, Oddo S (2007) Intracellular amyloid-beta in Alzheimer's disease. Nat Rev Neurosci 8:499-509.

Lewandoski M (2001) Conditional control of gene expression in the mouse. Nat Rev Genet 2:743-755.

Macdonald JL, Verster A, Berndt A, Roskams AJ (2010) MBD2 and MeCP2 regulate distinct transitions in the stage-specific differentiation of olfactory receptor neurons. Mol Cell Neurosci 44:55-67.

Nguyen MQ, Zhou Z, Marks CA, Ryba NJ, Belluscio L (2007) Prominent roles for odorant receptor coding sequences in allelic exclusion. Cell 131:1009-1017.

Oddo S, Caccamo A, Shepherd JD, Murphy MP, Golde TE, Kayed R, Metherate R, Mattson MP, Akbari Y, LaFerla FM (2003) Triple-transgenic model of Alzheimer's disease with plaques and tangles: intracellular Abeta and synaptic dysfunction. Neuron 39:409-421.

Reese TS (1965) Olfactory cilia in the frog. J Cell Biol 25:209-230.

Schwob JE, Szumowski KE, Stasky AA (1992) Olfactory sensory neurons are trophically dependent on the olfactory bulb for their prolonged survival. J Neurosci 12:3896-3919.

Su JH, Anderson AJ, Cummings BJ, Cotman CW (1994) Immunohistochemical evidence for apoptosis in Alzheimer's disease. Neuroreport 5:2529-2533.

Takahashi H, Brasnjevic I, Rutten BP, Van Der Kolk N, Perl DP, Bouras C, Steinbusch HW, Schmitz C, Hof PR, Dickstein DL (2010) Hippocampal interneuron loss in an APP/PS1 double mutant mouse and in Alzheimer's disease. Brain Struct Funct 214:145-160.

Talamo BR, Rudel R, Kosik KS, Lee VM, Neff S, Adelman L, Kauer JS (1989) Pathological changes in olfactory neurons in patients with Alzheimer's disease. Nature 337:736-739.

Tampellini D, Gouras GK (2010) Synapses, synaptic activity and intraneuronal abeta in Alzheimer's disease. Front Aging Neurosci 2:13.

Tao-Cheng JH, Dosemeci A, Winters CA, Reese TS (2006) Changes in the distribution of calcium calmodulin-dependent protein kinase II at the presynaptic bouton after depolarization. Brain Cell Biol 35:117-124.

Thinakaran G, Koo EH (2008) Amyloid precursor protein trafficking, processing, and function. J Biol Chem 283:29615-29619.

Troncoso JC, Sukhov RR, Kawas CH, Koliatsos VE (1996) In situ labeling of dying cortical neurons in normal aging and in Alzheimer's disease: correlations with senile plaques and disease progression. J Neuropathol Exp Neurol 55:1134-1142.

Vedin V, Molander M, Bohm S, Berghard A (2009) Regional differences in olfactory epithelial homeostasis in the adult mouse. J Comp Neurol 513:375-384.

Verhaagen J, Oestreicher AB, Gispen WH, Margolis FL (1989) The expression of the growth associated protein B50/GAP43 in the olfactory system of neonatal and adult rats. J Neurosci 9:683-691.

Wesson DW, Levy E, Nixon RA, Wilson DA (2010) Olfactory dysfunction correlates with amyloid-beta burden in an Alzheimer's disease mouse model. J Neurosci 30:505-514.

Wirths O, Bayer TA (2010) Neuron loss in transgenic mouse models of Alzheimer's disease. Int J Alzheimers Dis 2010:723782.

Wirths O, Multhaup G, Bayer TA (2004) A modified beta-amyloid hypothesis: intraneuronal accumulation of the beta-amyloid peptide- the first step of a fatal cascade. J Neurochem 91:513-520.

Yuan J, Yankner BA (2000) Apoptosis in the nervous system. Nature 407: 802-809. 